\title{
La relation entre le judiciaire et les victimes dans le contexte de la détermination de la peine au Canada : un enjeu pour l'évolution du droit criminel
}

\author{
Sébastien Labonté ${ }^{* *(\mathbb{D})}$
}

\begin{abstract}
Résumé
L'objectif de cet article est de divulguer des résultats de recherches exploratoires sur le rapport entre le système judiciaire canadien et les victimes d'actes criminels dans le contexte de la détermination de la peine. De manière plus précise, l'auteur s'intéresse au processus de mise en forme des attentes des victimes qui opère à ce stade des procédures judiciaires. Pour observer ces mises en forme, l'auteur s'appuie sur des décisions judiciaires qui ont été rendues par les tribunaux canadiens. À partir de son corpus empirique, l'auteur décrit comment certaines attentes victimaires sont plus audibles que d'autres et comment leur intégration dans le processus de détermination de la peine varie en fonction du type d'attente et des circonstances à l'intérieur desquelles elles émergent.
\end{abstract}

Mots-clés : Détermination de la peine, victimes, droit criminel, Évolution du droit criminel

\begin{abstract}
The purpose of this article is to disclose exploratory research findings on the relationship between the judicial system and victims of crime in the context of sentencing. More specifically, the author is interested in the process of formatting the expectations of the victims that operates at this stage of the judicial proceedings. In order to observe this phenomenon, the author relies on judicial decisions that have been rendered by Canadian courts. From his empirical corpus, the author describes how some victim expectations are more "audible" than others and how their integration into the sentencing process varies according to the type of expectations and the circumstances in which they emerge.
\end{abstract}

Keywords: Sentencing, Victims of crime, Criminal Law, Criminal Law Evolution

** L'auteur tient à remercier les évaluatedurs anonymes de même que l'équipe de la Revue pour leurs judicieux commentaires à l'égard de notre manuscrit.

Canadian Journal of Law and Society / Revue Canadienne Droit et Société, 2020,

Volume 35, no. 1, pp. 89-110. doi:10.1017/cls.2020.1 


\section{Introduction}

Dans le cadre de cet article, nous nous intéressons à la relation qu'entretient le système judiciaire avec les victimes d'actes criminels dans le contexte de la détermination de la peine. Bien que cette relation se soit développée un peu partout en Occident (Salas 2005; Kirchengast 2010; Cassel 2015), nos observations se limitent cependant à la situation canadienne.

Nous pouvons distinguer au moins deux modèles d'analyse pour étudier la relation entre le droit criminel et les victimes d'actes criminels. Le premier est le modèle qui domine actuellement les sciences sociales et qui consiste à étudier la relation à partir du point de vue des victimes. Dans cette perspective, on s'intéresse généralement aux expériences des victimes au sein des procédures judiciaires et à leur (in)satisfaction (Baril et collab. 1984; Wemmers et Raymond 2011; Rossi 2011; Lemonne 2011). Quant au deuxième modèle d'analyse, il est complémentaire au premier et se centre plutôt sur le système judiciaire et sur la manière dont celui-ci réagit face à la participation des victimes lors des audiences sur la détermination de la peine ${ }^{1}$. Les recherches privilégiant ce modèle d'analyse sont souvent des études sociojuridiques qui problématisent l'état du système judiciaire. Elles étudient le point de vue des acteurs judiciaires ${ }^{2}$ ou la manière dont ces derniers utilisent les informations fournies par les victimes ${ }^{3}$. Dans le cadre de cet article, c'est ce deuxième " modèle d'analyse " que nous privilégions. Nous proposons ainsi d'observer comment le système judiciaire va se positionner par rapport aux « inputs » victimaires au moment de déterminer la peine. Ces « inputs » victimaires sont évidemment pluriels et variés (Adam et collab, 2014). Ils peuvent apparaître sous la forme d'une description des torts subis comme ils peuvent prendre la forme d'un besoin, d'un souhait ou encore d'une exigence en matière de justice (Dubé et Garcia, 2017b). Nous pouvons également concevoir ces inputs comme des attentes, en l'occurrence des « attentes victimaires ${ }^{4}$ ».

Nous problématisons cette relation à l'intérieur de la thèse plus générale de la «non-évolution » du droit criminel qui provient de la théorie de la rationalité pénale moderne (RPM) (Pires 2002). Cette théorie fait dépendre les conditions d'évolution du droit criminel moderne de ces idées fondatrices qu'elle identifie au champ des normes de sanction (Dubé et Cauchie 2007, 471; Dubé et Labonté 2016, 688-689). Pires (1998) a identifié ces idées fondatrices comme étant celles des théories de la peine de rétribution, de dissuasion, de dénonciation et de réhabilitation carcérale (Dubé et Cauchie 2007, 472). Nous ne pouvons entrer

Mentionnons qu'il existe un large bassin d'études qui ont soulevé la relation entre les victimes et le système politique (p. ex. Elias 1986; Elias 1993; Garland 2001; Kaminski 2009).

Voir par exemple : Roberts et Edgar (2006).

Par exemple, voir : Roberts et Manikis 2010, Manikis 2015a; Manikis 2015b; Markin 2017; Labonté 2017).

Dans le cadre de cet article, nous employons le terme " victimaire ", non pas comme un substantif, mais comme un adjectif épithète. Par conséquent, par « attentes victimaires », nous renvoyons aux attentes des victimes incluant aussi bien celles valorisant la clémence que celles valorisant la répression. 
dans les détails dans le cadre de cet article, mais chacune de ces théories de la peine valorise l'affliction et/ou l'exclusion sociale du condamné (Ibid.). La théorie de la RPM établit une distinction entre le simple changement et l'évolution, et réserve cette dernière notion pour décrire un phénomène plus rare et plus substantiel qui implique l'observation de transformations innovatrices ${ }^{5}$ sur le plan des idées fondatrices du droit de punir. La thèse de la non-évolution défend tout simplement l'idée selon laquelle, en dépit des nombreux changements survenus au sein du droit criminel, ces changements n'ont pas eu pour effet d'apporter une transformation innovatrice sur le plan des normes de sanction ${ }^{6}$. C'est à l'intérieur de cette problématique de la non-évolution du droit criminel que s'inscrivent nos observations à l'endroit de la relation entre le système judiciaire et les victimes d'actes criminels, relation qui s'est développée à l'intérieur du champ des normes de sanction. Insistons sur le fait que notre attention se centre davantage sur le système judiciaire canadien que sur le système de droit criminel dans son ensemble. À l'heure actuelle, nous avons encore du mal à comprendre la portée de ce nouvel encadrement de la victime sur le processus de détermination de la peine du système judiciaire (Manikis 2015a; Manikis 2015b). Cauchie et Sauvageau (2013) ont pour leur part exploré ces questions sous l'angle $\mathrm{du}$ "closure ». Cette avenue mérite d'être explorée d'une manière plus approfondie qui ne se limiterait pas exclusivement à la question du closure, mais qui s'interrogerait plus globalement sur l'influence de ce nouveau rôle victimaire sur le choix de la sanction criminelle de même que sur l'évolution du droit criminel moderne.

Alors que certains n'ont pas hésité à dénoncer les dangers de ce rapprochement (Salas 2005; Erner 2006; Bensussan 2009; Quigley 2010), nous défendons plutôt la thèse que le danger résiderait moins dans le rapprochement en tant que tel que dans le fait qu'il se développe à l'intérieur du programme de la "rationalité pénale moderne " (RPM) (Pires 2001a; Parent 1995). En effet, que pourraient être les conséquences de ce rapprochement entre le système judiciaire et les victimes si celui-ci ne modifie pas son programme qui « (sur)valorise l'affliction et l'exclusion sociale » du coupable (Garcia 2013)?

Cet article contient trois parties. D'abord, nous allons présenter quelques éléments du contexte canadien ayant favorisé le développement de la relation entre le système judiciaire et les victimes dans le processus de détermination de la peine. La deuxième partie portera sur la méthodologie. Les résultats de recherche seront présentés dans la troisième partie.

5 Les concepts de redondance et d'innovation sont compris, l'un et l'autre, comme étant réciproquement le contre-concept de l'autre. L'innovation se caractérise par l'opposition à la redondance. À l'intérieur de la théorie de la RPM, l'innovation s'oppose alors aux valeurs de l'affliction et de l'exclusion sociale du condamné.

6 Il faut faire preuve de prudence. La théorie de la RPM reconnaît que des systèmes d'idées innovatrices - comme la justice restauratrice par exemple - gravitent dans l'univers cognitif du droit criminel moderne, mais elle reconnaît également que ces derniers se retrouvent marginalisés par la domination de la rationalité pénale moderne (Dubé et Garcia 2017, 3). 


\section{Mise en contexte : l'inclusion de la victime dans le champ des normes de sanction}

Parmi les lois fédérales ayant contribué significativement à l'inclusion de la victime dans les normes de sanctions, nous retrouvons le projet de loi C-89 ${ }^{7}$ qui, en introduisant la déclaration de la victime, étendait la participation des victimes dans les procédures judiciaires au processus de détermination de la peine (Manikis $2015 \mathrm{~b}, 173)^{8}$. Cette déclaration autorise la victime à introduire, lors des audiences sur la peine, de l'information concernant l'étendue des conséquences que l'infraction a eues sur elle ${ }^{9}$. Au Canada, la déclaration de la victime fit son arrivée en $1988^{10}$.

Dans la première mouture de la déclaration de la victime, le tribunal pouvait prendre en considération ces inputs victimaires au moment de déterminer la peine, mais sans avoir une obligation juridique de le faire (Grossman et Kane 2008, 290; Katz 2010, 1-2; Manikis 2015a, 87). Toutefois, un amendement en 1995 a fait en sorte que le tribunal soit désormais dans l'obligation de " considérer » lesdits inputs (Katz 2010, 2). Comme le souligne à juste titre Manikis (2015a 86), le Code criminel n'offre pas de directive sur la manière dont ces inputs doivent être considérés ${ }^{11}$. Plus tard, en 1999, un autre amendement ${ }^{12}$ a en outre permis aux victimes de présenter leur déclaration oralement devant le tribunal (ibid.). Selon Verdun-Jones et Tijerino $(2004,472)$, la permission accordée aux victimes de soumettre oralement leur déclaration représentait l'une des modifications les plus « dramatiques » de celles apportées par cet amendement.

Ensuite, en 1996 , le projet de loi C- $41^{13}$ a ajouté deux objectifs à la peine concernant directement les victimes d'actes criminels : réparer les torts causés à la victime et susciter, chez le contrevenant, la reconnaissance des torts causés. La codification de ces objectifs ne garantit pas que les intérêts des victimes seront pris en compte, mais elle rend cette éventualité probable.

Finalement, l'adoption du projet de loi C- $32^{14}$, en juillet 2015 , a contribué elle aussi au développement de la relation entre le droit criminel et les victimes. Ce

P.L. C-89, Loi modifiant le Code criminel (victimes d'actes criminels), $2^{\mathrm{e}}$ session, $34^{\mathrm{e}}$ Parlement, 1989, art. 735 (sanctionné en décembre 1989).

8 Il faut insister ici sur le fait que les victimes ont traditionnellement été cantonnées au rôle de témoins et par conséquent, ont été traditionnellement exclues du processus de détermination de la peine (Roach 1999, 290). L'introduction de la déclaration de la victime rompt avec cette tradition en lui permettant de participer au sein du processus de détermination de la peine.

9 La Cour de l'Ontario précisait les balises de la déclaration de la victime : « Impact statements should describe "the harm done to, or loss suffered by, the victim arising from the commission of the offence". The statements should not contain criticisms of the offender, assertions as to the facts of the offence, or recommendations as to the severity of punishment. » (R. v. Gabriel, 1999, par. 29).

10 Précisons que la prise en compte des conséquences du crime pouvait se faire bien avant l'introduction de la déclaration des victimes, notamment par l'intermédiaire du procureur de la couronne et des rapports présentenciels (Dubé et Garcia, 2018).

11 Le présent article se donne justement l'objectif d'observer comment les tribunaux canadiens ont " considéré » (ou non) certains inputs victimaires.

12 P.L. C-79, Loi modifiant le Code criminel (victimes d'actes criminels) et autres lois en conséquence, $1^{\text {ère }}$ session, $36^{\mathrm{e}}$ Parlement, 1999, art. 722 (sanctionnée le 17 juin 1999).

13 PL C-41, Loi modifiant le Code criminel (détermination de la peine) et d'autres lois en conséquence, $1^{\text {ère }}$ session, $35^{\mathrm{e}}$ Parlement, 1995 (sanctionné le 13 juin 1995).

14 P.L. C-32, Loi édictant la Charte canadienne des droits des victimes et modifiant certaines lois, $41^{\mathrm{e}}$ législature, $2^{\mathrm{e}}$ session, 2015 (sanctionné le 23 avril 2015). 
projet de loi possède deux volets : l'avènement de la Charte canadienne des droits des victimes $^{15}$ et des amendements au Code criminel. La Charte des victimes introduit une rupture par rapport au statut légal des victimes : celles-ci ne sont plus seulement de simples témoins, mais bien des individus possédant des intérêts légitimes et des droits (Perrin 2017, 23). Parmi les amendements au Code criminel qu'introduit le projet de loi C-32, il faut mentionner l'élargissement de l'objectif de la dénonciation ${ }^{16}$ pour y inclure la dénonciation des torts subis par les victimes. Selon cet objectif mis au goût du jour, la peine n'a plus pour but de seulement dénoncer le caractère illégal de l'acte condamnable, elle dénonce désormais aussi les torts subis par la victime.

Toute cette activité législative canadienne a eu pour effets de franchir un point de non-retour au niveau de la relation qui nous intéresse : il ne fait nul doute que le rôle de la victime eût été élargi de manière à lui permettre de participer aux procédures judiciaires lors de la détermination de la peine ${ }^{17}$.

Nous pouvons dire qu'au cours des dernières décennies, le rôle de la victime est passé de celui de "victime-témoin de l'acte " à celui de "victime-témoin de l'impact ${ }^{18}$ contre sa propre personne. Cette transition souligne au moins deux phénomènes distincts. D'abord, il indique l'entrée des victimes d'actes criminels au sein d'un champ normatif qui leur était inaccessible, soit le champ des normes de sanctions (les normes qui sont sollicitées pour la gestion de la peine). Ensuite, fort de cette inclusion récente au sein des normes de sanctions, ce passage de la victimetémoin de l'acte à la victime-témoin de l'impact renvoie à l'idée d'un élargissement de la définition juridique de la victime pour y inclure non seulement les proches des victimes, mais aussi la collectivité ${ }^{19}$.

Cette situation contraste avec la tradition d'un droit criminel qui s'est historiquement construite sans la " participation impliquée » des victimes à ce moment particulier des procédures (Labonté 2017). La question qui nous interpelle dans cet article est celle de savoir quelle place le système judiciaire va faire à la victime dans le processus de détermination de la peine.

\section{Méthodologie : une analyse documentaire à partir de la jurisprudence canadienne}

Sur le plan de la méthode, nous avons privilégié une analyse documentaire. Nous avons observé le rapport entre le système judiciaire et les victimes exclusivement à partir de décisions judiciaires qui ont été rendues au cours des trois dernières décennies par les tribunaux canadiens. Nous avons vu dans ce type de document

15 Désormais, nous nous y référerons en tant que Charte des victimes.

16 art. 718(a), C. cr.)

17 Dans les décisions judiciaires récentes, il n'est désormais plus rare d'observer une rubrique réservée aux victimes et à la communauté dans laquelle le tribunal évalue les inputs victimaires et y réagit.

18 Nous tenons à remercier Alvaro P. Pires pour la suggestion de cette distinction.

19 Notamment à travers les Community Impact Statement qui ont été introduits en 2011 et circonscrits initialement aux infractions de fraude. En 2015, le projet de loi C-32 a élargi le contexte d'application des Community Impact Statement à toutes les infractions. Dans le cadre de notre article, nous ne pouvons pas adresser la prise en compte de ces déclarations. 
un lieu d'observation idéal pour étudier notre objet de recherche ${ }^{20}$. Pour constituer notre corpus empirique, nous disposions virtuellement de l'ensemble des décisions judiciaires canadiennes traitant de la matière criminelle et pénale ${ }^{21}$. Dans un va-et-vient continu entre l'empirie et l'analyse, nous avons progressivement établi un univers de travail constitué de 331 décisions, mais nous avons procédé seulement à l'analyse de 95 décisions, ce qui représente notre corpus empirique $^{22}$. Nous avons pu repérer ces décisions grâce à des moteurs de recherche tels que Nexis Lexis Quicklaw et CanLii ${ }^{\oplus}$. Nous pouvons dire de

20 Dans le cadre de notre recherche, la documentation jurisprudentielle prend la forme de communications juridiques stabilisées laissant des traces empiriques sur la manière dont le système judiciaire observe le monde et la manière dont il s'auto-observe. Puisque le rapprochement entre le SDC et les victimes relève d'un phénomène relativement récent, il est probable que ce système social, dans le traitement des affaires judiciaires, en soit encore à l'étape de l'élaboration des principes, ce qui fait normalement couler plus d'encre qu'une décision prise en vertu d'un vieux principe déjà bien stabilisé dans l'état du droit.

21 Toutefois, il convient de préciser que toutes les décisions rendues ne sont pas accessibles. Des décisions sont rendues sans qu'elles soient transcrites à l'écrit, ce qui rend leur analyse impossible à l'intérieur de notre dispositif de recherche. Cependant, cette perte de matériel empirique entraîne peu ou pas de conséquence à l'égard de notre projet de recherche. Effectivement, si ces décisions ne sont pas stabilisées ou matérialisées sous forme écrite, c'est parce que le système judiciaire luimême estime qu'elles ne constituent pas un enjeu majeur du point de vue du droit. Autrement dit, les décisions écrites sont celles que le système sélectionne pour rendre compte de l'état du droit. R. v. Gabriel, 1999 O.J. 2579; R. v. Bullen, 2001 YKTC 504; R. c. Dufour, 2015 J.Q. 6385; R. v. J.W., 2016 O.J. 5178; R. c. Chav, 2011 QCCQ 10468; R. c. Blondin, 1995 J.Q. 2786; R. c. Gatambwe, 2013 QCCQ 5749; DPCP c. Tétrault-Hamel, 2015 QCCQ; R. c. Ramla, 2016 J.Q. 3070; R. c. Zelensky, 1978 2. R.C.S. 940; R. c. Tremblay, 1998 CanLii 9248 (QC CQ); R. c. Charpentier, 2015 J.Q. 2897; R. c. Godin, 2016 J.Q. 8074; R. v. D.B., 2016 A.J. 92; R. c. McDonald, 2016 J.Q. 6642; McCraw c. R., 19913 R.C.S. 83d; R. c. Fréchette, 2016 JQ 1198; R. c. Martin, 2012 JQ 4819; R. c. Martel, 2016 JQ 11001; R. c. C.C., 2018 QCCQ 3610; R. c. M.-L., 2018 QCCQ 1586; R. c.P.F., 2011 QCCQ2236; Paul c. R., 2012 QCCA 172; R. c. Landry, 2013 QCCQ 3013; R. v. J.S.M., 2016 N.S.J. 493; R. v. Millington, 2015 BCSC 1612 (CanLii); R. c. P.F., 2011 QCCQ 2236; R. c. Belleau, 2010 QCCQ 3407; R. c. M.L., 2018 QCCQ 1586 (CanLII); R. c. Belfoy, 2018 QCCQ 3025 (CanLII); R. c. Alonso, 2012 QCCQ 7919 (CanLII); R c Didier, 2005 CanLII 56812 (QC CQ); R. c. Malmo-Levine, 2003 R.C.S. 694 323; R. c. Kapanga, 2015 QCCM 106 (CanLii); R. c. C.C., 2018 QCCQ 3610; R. c. M.(C.A.), 19961 R.C.S. 500; R. v. Morgan, 2016 canlii 60965 (NLPC); R. c. Guértin, 2016 QCCQ 11256; R. v. Nicholls, 2015 ONSC 8136; R. v. Mooswa, 2004 S.J. 851; R. c. Guay, 2005 CanLii 33319 (QCCQ); R. v. H.V., 1998 O.J. 4694; R. c. Brutus, 2009 QCCA (CanLii); R. c. Tessier, 2005 CanLii 15013 (QCCQ); R. $c$. Chicoine, 1999 CanLii 10548 (QC CQ); R. c. Michaud, 2009 QCCQ 5109 (CanLii); Michaud c. R., 2010 QCCA 152; R. c. Perry, 2011 J.Q. 2709; R. c. Deschênes, 2012 J.Q. 12755; R. c. S.T., 2017 QCCQ 8801; R. c. M.S., 2005 NBBR 41 (Canlii); R. c. A.R., 2004 J.Q. 10754; R. c. Proulx, 20001 R.C.S. 61; R. c. Leblanc, 2013 QCCS 753 (CanLii); R. v. C.D.C., 2004 CanLii 19836 (NLPC); R. v. J.L., 2015 BCPC 316 (CanLii); R. c. Nadeau, 2009 QCCQ 1156 (CanLii); R. v. J.F., 2001 O.J. 2054 (QL); R. v. Cassidy, 2016 ONSC 3765 (CanLii); R. v. Van Eindhoven, 2007 NUVCJ 02; R. c. Fitzgibbon, 1990 1 R.C.S. 1005; R. c. Ford, 2002, Canlii 34585 (QC CQ); LSJPA-1631, 2016 QCCQ 9175; R. c. ColeSmith, 2017 A.N.-B. 154; R. c. Simoneau, 2017 QCCA 1382; R. v. Denny, 2016 NSSC 76 (Canlii); R. c. Jordan, 20161 S.C.R. 631; R. c. Courtemanche-Brisebois, 2006 QCCQ 12494 (CanLii); R. c. Cedar, 2011 Canlii QCCQ 14965; R. v. J.C., 2011 MBPC 71 (CanLii); R. c. M.G., 2010 J.Q. 525; R. c. M.G., 2009 QCCQ 12940 (Canlii); R. c. E.F., 2016 JQ 5808; DPCP c. Girard-Lévesque, 2016 QCCQ 7602; R. c. Gignac, 2016 JQ 5767; R. c. Rancourt, 2016 JQ 11424; R. c. L.L., 2009 JQ 5952 324; R. c. Brouillette, [1988] J.Q. no 315; R. c. Turcotte, 2016 QCCS 112; R. c. Larendeau, 2007, QCCQ 1593 (CanLii); R. c. Regis-Fodé, 2015 QCCQ 8160 (CanLII); R. c. Fortin, 2010 QCCQ 5727 (CanLII); R. c. S.G., 2010 QCCQ 2651 (CanLII); R. c. Benoit, 2010 QCCQ 1707 (CanLII); R. c. Choukroun, 2017 QCCQ 1284 (CanLII); R. c. Lacasse, 2015 QCCQ 1367 (CanLII); R. c. Amelaine, 2018 QCCQ 8533 (CanLII); R. c. Vernier, 2015 QCCQ 12899; R. c. Elfaf, 2007 QCCQ 17172 (Canlii); R. v. BasilBorutski, 2017 ONSC 89769 (CanLii); R. c. Tremblay, 2010 QCCQ 2856 (Canlii); Kubala c. R., 2017 QCCA 882, par. 13-14 
manière globale que la sélection des décisions judiciaires a pris la forme d'une « étude collective de cas » (Stake 1994, cité par Pires 1997, 152), c'est-à-dire que nous avons cherché à trouver des décisions qui jetaient de la lumière sur différents aspects du phénomène étudié de façon à établir une « mosaïque par l'entremise d'un nombre diversifié de cas " (Pires 1997, 157). En abordant le processus de sélection de manière exploratoire, nous avons pu identifier progressivement les contextes ou les situations dans lesquelles les références aux victimes étaient susceptibles de se manifester dans les décisions judiciaires. Une fois identifiés les situations ou les contextes pertinents, nous avons dès lors adopté une logique "d'échantillonnage par contraste-approfondissement ${ }^{23}$ " (Pires 1997, 160). Cette stratégie d'échantillonnage nous a été très utile lorsque nous avons trouvé des décisions dans lesquelles les victimes adoptaient une attitude punitive. Nous avons alors délibérément cherché des décisions dans lesquelles les victimes adoptaient une attitude inverse en prônant le pardon, la clémence et la modération. Spécifions que l'objectif théorique était moins de comparer les attitudes adoptées par les victimes que la réaction des tribunaux à leur égard.

Pour qu'une décision soit intégrée au corpus empirique, il fallait évidemment qu'elle effectue des renvois aux victimes et que ces derniers fournissent suffisamment de détails pour observer, d'une part, les inputs victimaires, et d'autre part, la réception de ces inputs par les tribunaux. Ces conditions étaient remplies lorsque le tribunal citait des passages de déclarations des victimes (orales ou écrites) in extenso ou encore lorsque les décisions prenaient la forme d'un verbatim de l'audience sur la détermination de la peine. Dans ce dernier cas, l'observation intégrale du discours des victimes lors des audiences sur la peine et leur réception par les tribunaux était possible.

Par rapport à l'analyse des inputs des victimes, nous avons procédé à une analyse du discours général (Duchastel 1993). Notre stratégie d'analyse comportait deux moments. Dans un premier temps, nous avons entrepris de décrire la variété des inputs victimaires que nous pouvions observer à partir de notre corpus empirique. Contrairement aux études centrées sur les victimes (tel que discuté dans l'introduction), cette étape ne constitue pas la finalité de notre recherche, mais plutôt une étape préliminaire à l'observation de la mise en forme juridique des attentes victimaires. Ce n'est que dans un deuxième temps que nous pouvions observer les formes que prennent ces inputs une fois transposés (ou non) dans le processus de détermination de la peine. En procédant ainsi, nous nous sommes donné les conditions nécessaires pour mettre en évidence le rôle actif du système judiciaire dans l'établissement de sa frontière entre les inputs admissibles et les inputs inadmissibles ou plus difficilement admissibles en matière de détermination de la peine. L'analyse qui suit présente les énoncés qui nous semblent être les plus représentatifs de ce que nous avons pu observer dans l'ensemble du corpus empirique.

23 Nous n'avons pas adopté l'ensemble de ce devis d'échantillonnage, mais seulement quelques aspects, notamment la recherche délibérée d'une situation ou d'un contexte faisant contraste à celles que nous avions déjà étudiée. 


\section{La réception inégale des « inputs » victimaires par le système judiciaire canadien}

Si le cadre législatif amène les victimes à se prononcer, dans leur déclaration, strictement sur les conséquences du crime, la loi n'établit pas explicitement de limites au contenu des déclarations des victimes. Ce sont plutôt les tribunaux qui se sont dotés de limites :

The law is clear. A victim may address the specific harm suffered, but not express either their views about the offender or about the sentence [...] This limitation is imposed not by legislation, but by the courts. The Criminal Code, in s. 722, describes the procedures for completing a victim impact statement. Nothing in these provisions precludes or provides for any further victim involvement ${ }^{24}$.

Les tribunaux se sont dotés d'une limite qu'ils ne doivent pas franchir, sauf dans certaines situations d'exception $^{25}$. Cette limite, nous allons tenter de l'illustrer à partir de nos observations empiriques sur la réception des attentes victimaires. Parmi les inputs victimaires, nous retrouvons ceux qui concernent la description des conséquences du crime sur les victimes, c'est-à-dire les torts subis par elles (3.1.). Nous incluons également les attentes de dédommagement (3.2.), les attentes de quiétude et de protection (3.3.). Nous avons pu identifier les recommandations des victimes sur la peine (3.4.) et les attentes de closure (nous en préciserons le sens supra) (3.5.). Ces attentes peuvent aller vers la répression ou vers la clémence.

Dans ce qui suit, nous allons présenter comment chacune de ces attentes tend à se transposer dans le contexte de la détermination de la peine.

\subsection{Les torts subis par les victimes et l'évaluation de la gravité de l'infraction}

Ces « inputs » sur les torts causés aux victimes sont surtout mobilisés par le tribunal pour déterminer la gravité de l'infraction qui a été commise ${ }^{26}$. En droit criminel canadien, la gravité de l'infraction constitue l'un des deux pôles du principe de justice fondamental de la proportionnalité ${ }^{27}$. Cela signifie que la "gravité de l'infraction " joue un rôle important dans le choix de la sanction (autant vis-à-vis de sa nature que de son quantum).

Les conséquences du crime sur la victime se rapportent à la dimension matérielle de l'infraction et les déclarations des victimes constituent un moyen utile pour les évaluer (Manikis 2015a, 95; Markin 2017, 105). Nous l'avons observé empiriquement à partir de la jurisprudence. En effet, l'utilité et l'importance des conséquences du crime dans l'évaluation de la gravité de l'infraction ont été réitérées à multiples reprises par les tribunaux canadiens ${ }^{28}$. Par exemple, la Cour

R. v. Bullen, 2001 YKTC 504, par. 34

Nous reviendrons sur ces exceptions.

La Cour suprême du Canada argumentait dans ce sens dans Malmo-Levine (2003, par. 235).

À l'article 718.1, le Code criminel canadien stipule que : « La peine est proportionnelle à la gravité de

l'infraction et au degré de responsabilité du délinquant " (Art. 718.1, C. cr.).

28 Voir R. c. Malmo-Levine, 2003 S.C.C. 74 (Canlii); R. v. Hammermeister, 2016 A.J. 1045, par. 30; R. v. Hajar, 2016 A.J. 754, par. 61-65. 
de la Nouvelle-Écosse, dans une affaire d'agression sexuelle d'un père de famille à l'endroit de l'amie (voisine) de sa propre fille, le tribunal a fait valoir que : " the impact on the victim is important in assessing how grievous of the offences are ${ }^{29}$. Lorsque les tribunaux déterminent la gravité de l'infraction, ils effectuent des sélections à partir d'au moins trois bassins d'information : un bassin d'éléments factuels sur les circonstances de l'infraction ${ }^{30}$; un bassin d'éléments jurisprudentiels et un bassin d'éléments légaux où les lois $^{31}$ et la volonté du Législateur ${ }^{32}$ sont interprétées.

La mobilisation des déclarations des victimes dans l'évaluation de la gravité de l'infraction constitue, selon les juges canadiens interviewés par Dubé et Garcia (2018), la limite de l'intégration des victimes dans le processus de détermination de la peine. Par conséquent, lorsque les tribunaux affirment « avoir pris en considération la déclaration des victimes ou avoir reconnu les torts subis dans la détermination de la peine ", il faut comprendre que ces torts ont été mobilisés dans l'évaluation de la gravité de l'infraction (Ibid.).

Nos observations empiriques confirment les observations faites par les autres chercheurs. Toutefois, notre analyse de la jurisprudence nous indique que l'intégration des victimes dans la détermination de la peine ne se limite pas seulement à l'évaluation de la gravité de l'infraction. L'intégration des victimes à ce moment des procédures s'effectue également sous d'autres thèmes.

\subsection{La réception des attentes de dédommagement}

Comme pour les inputs précédents, les demandes de dédommagement n'éprouvent aucune difficulté à se transposer en information juridiquement pertinente pour déterminer la peine. Par contre, là où le bât blesse, c'est dans la mise en application des ordonnances de dédommagement, c'est-à-dire leur imposition (Young 2001, 22). Le critère des "dommages facilement calculables ${ }^{33}$ " agit comme un obstacle majeur à l'imposition d'ordonnances de dédommagement. Il suffit d'imaginer une victime voulant obtenir un dédommagement pour poursuivre une thérapie. Comment déterminer la valeur des torts psychologiques subis par une victime? En déterminant le nombre de séances de thérapie nécessaires? Comment déterminer le nombre de séances nécessaire? Cet exemple n'est pas fictif, il provient de la décision LSJPA-1631 $1^{34}$. Comme, dans cette affaire, la valeur du « dommage » n'était pas

29 R. v. J.S.M., 2016 N.S.J. 493, par. 62.

30 Ce bassin regroupe des faits épars que nous ne pouvons pas décrire ici. Ils peuvent concerner autant la situation de la victime que celle de l'accusé. Concernant la victime, on tient compte des torts qu'elle a subis, mais aussi de son lien social avec l'accusé (parent-enfant; professeur-élève; conjointconjointe, etc.). L'âge de la victime et sa vulnérabilité entrent aussi en ligne de compte. Ce bassin d'éléments factuels est surtout mobilisé lors de l'appréciation de la gravité subjective et, dans bien des dossiers, son appréciation s'effectue exclusivement à partir des torts subis par la victime.

31 Par exemple, les procédures prévues (sommaire ou par acte criminel), les peines minimales et les peines maximales sont interprétées comme des indicateurs légaux de la gravité d'une infraction donnée (Boisvert et Jodouin, 2002).

32 Par exemple, lorsqu'un amendement modifie la loi de sorte à augmenter les peines maximales, les tribunaux interprètent généralement cette hausse des peines maximales comme un message du Législateur communiquant la gravité d'une infraction donnée.

33 (Art. 738.1(a) à (e), C. cr.)

34 LSJPA-1631, 2016 QCCQ 9175. 
facilement calculable, le tribunal n'a pas pu prononcer le dédommagement demandé par la victime ${ }^{35}$. Précisons que le tribunal n'a éprouvé aucune difficulté à comprendre la requête de la victime et qu'il s'est même montré réceptif à sa situation.

La présence de ce critère est au cœur de l'enjeu de la différenciation entre le droit civil et le droit criminel. Pour maintenir une frontière entre les tribunaux criminels et civils, les cours de justice criminelles évitent de se "civiliser " au sens de se substituer aux tribunaux civils. ${ }^{36}$ Cela explique en partie pourquoi le dédommagement n'a pas le statut d'une sanction autonome en droit criminel canadien, sinon les tribunaux criminels devraient nécessairement s'adonner à des calculs complexes pour déterminer la valeur du dommage. Les énoncés suivants illustrent cette préoccupation des tribunaux criminels :

Les tribunaux criminels ne doivent pas se substituer aux tribunaux civils et le processus de détermination de la peine n'est jamais le bon forum pour établir des dommages sérieusement contestés ou juridiquement plus complexes $[\ldots]^{37}$.

Je ne crois pas que le législateur, à l'article 738 C.cr., ait voulu confier à des juges exerçant en matière criminelle le soin de décider de litiges civils dépassant le million de dollars. C'est d'ailleurs l'esprit des décisions de la Cour suprême du Canada citées plus haut $[\ldots]^{38}$.

Ce qu'il faut comprendre à partir de ces énoncés, c'est que l'autoportrait punitif $\mathrm{du}$ droit criminel agit comme un obstacle, non pas au niveau de la réception des attentes de dédommagement, mais au niveau de l'application des ordonnances de dédommagement, en limitant le dédommagement à une simple ordonnance facultative plutôt qu'à une sanction autonome en droit criminel (Pires et Acosta 1994, 20-21). Sous l'influence d'un programme plus «citoyen » et non plus sous celui de la RPM, le droit criminel aurait plus de facilité à concevoir le dédommagement comme un moyen suffisant de résoudre un conflit, sans y voir une ingérence du civil dans le criminel ou une dédifférenciation entre le civil et le criminel. Comme le programme de la RPM insiste sur les normes de sanctions pour construire son identité et qu'il valorise l'affliction et l'exclusion sociale du coupable, le dédommagement n'apparaît pas comme une sanction suffisamment forte pour réitérer cette identité guerrière (Pires 1990). Dans un autre système d'idées, plus « citoyen » que « guerrier ", l'identité pourrait se fonder non plus sur les normes de sanction, mais plutôt sur les normes de comportement, c'est-à-dire les illicites définis comme étant criminels. En construisant son identité sur les normes de comportement, le droit criminel pourrait valoriser le dédommagement et en faire une sanction autonome sans dissonance à l'égard de son identité systémique. Par ailleurs, sous un tel programme plus « citoyen », le critère du « dommage facilement calculable » pourrait prendre une autre forme. Plutôt que d'exiger que la valeur des

\footnotetext{
35 Voir aussi R. c. Tremblay, 1998 CanLii 9248 (QC CQ), par. 47; R. c. Cole-Smith, 2017 NBBR 96, par. 21-22;

36 À cet égard, voir la décision Zelensky (1978) de la Cour Suprême du Canada à la page 963.

R. c. Simoneau, 2017, QCCA 1832, par. 36.

R. c. Ford, 2002, Canlii 34585 (QC CQ), par. 17
} 
dommages soit facilement calculable en vue d'ordonner le versement d'une somme proportionnelle au dommage, les tribunaux pourraient stipuler comme peine n'importe quel montant, plus élevé ou moins élevé que les dommages civils et adapté au revenu du coupable. Par la suite, si la victime estime que le montant est insatisfaisant, elle pourrait aller au tribunal civil pour recevoir la différence (selon les calculs du tribunal civil). Cette interprétation est rendue difficile, voire complètement " invisibilisée » par la domination actuelle du programme de la RPM qui dévalorise le dédommagement comme mode de résolution de conflit (Pires 1990) et qui rend l'exigence d'un dédommagement proportionnel au dommage inflexible quitte à se coincer dans une logique du « ça passe ou ça casse ». Le principe de proportionnalité agit ici, dans le cas du dédommagement, comme un obstacle à la mise en œuvre des ordonnances.

\subsection{La réception des attentes de quiétude et de protection}

Les attentes de quiétude et de protection représentent deux types d'attentes distinctes l'une de l'autre, mais que nous traitons sous un même point puisqu'elles se transposent sensiblement de la même manière dans le processus de détermination de la peine. Les attentes de quiétude concernent l'intégrité psychique de la victime alors que les attentes de protection concernent plutôt leur intégrité physique. Nous pouvons nous les représenter comme étant les deux aspects d'un même problème de "sécurité ".

Si l'infraction commise est poursuivie par procédures sommaires, les demandes de quiétude et de protection des victimes se transposent en des interdictions de communications et de contacts entre l'accusé et la victime ${ }^{39}$. Lorsque l'infraction commise est poursuivie par voie de mise en accusation criminelle, ces demandes peuvent être sélectionnées, entre autres, pour justifier une peine d'emprisonnement. Lorsque cette peine d'emprisonnement est suivie d'une ordonnance de probation, les interdictions susmentionnées sont également incluses. De la sorte, le tribunal estime assurer une protection de la victime par l'incarcération, laquelle se prolonge par les interdictions de communications et de contacts. Dans la décision A.R., la protection d'une victime de violence conjugale constitue le souci primordial du tribunal, si bien qu'il met le principe de proportionnalité en arrière-plan pour favoriser la protection de la victime :

[...] le Code criminel actuel ne permet pas au juge qui rend une ordonnance de détention supérieure à deux ans de prononcer des interdictions de contact, si essentielles à la protection et au besoin de sécuriser les victimes. Cela m'apparaît particulièrement malheureux dans le dossier sous étude. [...] La Cour ne peut se résoudre à imposer la peine juste et appropriée de 26 mois de détention puisqu'elle considère que la protection dont doit jouir la victime vaut plus que quelques semaines de détention additionnelles. La peine sera donc ajustée à deux ans de détention à la seule fin d'ajouter une

39 Il est à noter que ces attentes peuvent également être traitées en amont et en aval des audiences de détermination de la peine, notamment par les décisions relatives à la caution de l'accusé et lors des décisions de mises en libération conditionnelle. Nous nous limitons ici à traiter de ces attentes dans le contexte de la détermination de la peine seulement. 
ordonnance de probation contenant les ordonnances d'interdiction et tant qu'à faire, de traitement. Cette peine de 2 ans d'emprisonnement assortie d'une ordonnance de probation de 3 ans, est ordonnée dans le deuxième dossier ${ }^{40}$.

Dans Marier, un autre dossier de violence conjugale, la victime manifeste son désir de "pouvoir retrouver la paix et la quiétude de sa vie passée ${ }^{41}$. Le tribunal impose une ordonnance de non-communication à l'accusé. Ces ordonnances de non-contact et de non-communication sont reconnues par les tribunaux comme étant des moyens par lesquels ces derniers peuvent offrir une certaine tranquillité d'esprit, une certaine quiétude, voire une forme de protection aux victimes ${ }^{42}$.

Les attentes de quiétude et de protection ne rencontrent pas d'obstacle une fois qu'elles ont été injectées dans le système de justice criminelle ${ }^{43}$. Ce sont des attentes qui peuvent aisément être prises en considération par les tribunaux sans ébranler « l'autoportrait punitif » du système (Pires 2001a, 184). Nous pouvons déduire que ces attentes de quiétude et de protection constituent de l'information juridiquement pertinente pour les tribunaux, et que ces derniers peuvent combler ces attentes victimaires en ajustant la sanction en conséquence.

\subsection{La réception des recommandations des victimes sur la peine}

Les recommandations des victimes sur la peine peuvent aller de la répression à la clémence/modération. En ce qui concerne les demandes de répression, le problème est assez complexe. À certaines occasions, les tribunaux peuvent en venir à associer ces demandes à une forme de vengeance alors qu'à d'autres occasions, ces demandes peuvent être perçues comme un indicateur de la répercussion de l'infraction sur les victimes. Lorsque ces demandes sont observées comme une forme de vengeance, elles sont évacuées du processus, comme c'est le cas dans l'énoncé suivant :

In the case before the court, it is clear that the family, friends and community of friends of [the victim] are very sad and angry and grieving. However, to the extent that the victim impact statements speak to a wish for an increased penalty, or even go so far as to express a desire for vengeance, they are not appropriate and have been disregarded by this court ${ }^{44}$.

\section{R. c. A.R., 2004, par. 34 et 37. \\ R. c. Marier, 2013 QCCS 3398 (canlii), par. 40).}

Dans la décision R. v. C.D.C., 2004, le tribunal indique que : " [in the context of probation orders] [...] such a condition [no contact and no communication order] is designed, in part, to provide a victim with protection and peace of mind. It is important that we not forget that even the slightest contact by an offender with his or her victim after an offence has been committed can be a deeply disturbing, troubling and intimidating occurrence for some victims of crime. » (R. v. C.D.C., 2004 19836 (NL PC), par. 37).

43 Dans R. c. Gatambwe, 2013 QCCQ 5749, par. 2, « La poursuite propose une absolution conditionnelle assortie de conditions pour assurer la protection de la victime. [...] ". Dans la décision DPCP c. Tétrault-Hamel, 2015 QCCQ 13768, par. 22, c'est l'agent de probation qui soulève le thème de la protection de la victime : "L'agent de probation est d'avis que pour ne pas compromettre la sécurité de la victime et de ses proches, il est nécessaire qu'une distance s'installe entre l'accusé et la victime». Voir entre autres: R. c. Ramla, 2016 J.Q. 3070, par. 105; R. v. Gabriel, 1999 J.Q. 2579, par. 10; R. c. Blondin, 1995 J.Q. 2786, par. 9.

$44 \quad$ R. v. Nicholls, 2015, p. 16. 
Cela dit, si les tribunaux évacuent ces inputs répressifs, ce n’est peut-être pas dû à un manque de soutien cognitif pour valoriser l'affliction et l'exclusion sociale, mais plutôt à la menace ${ }^{45}$ que représentent les victimes répressives pour l'intégrité du processus de détermination de la peine. Dans l'affaire Mooswa, les parents de victimes décédées dans une infraction de conduite en état d'ébriété ont suggéré au juge de lourdes peines d'incarcération, plus lourdes que celle qui avait été soumise conjointement par les parties. Le juge a pris position et a accepté la soumission conjointe de dix ans d'emprisonnement, et a ainsi prononcé la peine la plus lourde permise par la jurisprudence de la province de la Saskatchewan. Au premier regard, on a l'impression qu'il ignore les proches des victimes, mais il s'adresse à elles en précisant qu'il n'a pas pu aller au-delà de cette peine en raison d'une contrainte sociojuridique (Dubé 2017) : la fourchette des peines établies par la jurisprudence. Autrement dit, le juge a donné aux parents des victimes ce qu'ils voulaient, mais avec les moyens qu'il avait à sa disposition. Par contre, cela ne signifie pas pour autant que la vengeance puisse constituer un motif légitime pour punir. La peine d'emprisonnement de 10 ans a été motivée par la rétribution et la dissuasion. La vengeance, en tant que motif pour punir, n'est juridiquement pas recevable ${ }^{46}$.

Lorsque les demandes de répression des victimes ne sont pas observées comme une forme de vengeance, ces demandes de peines sévères sont perçues comme un indicateur du degré de leur souffrance et comme une indication de la gravité du crime qui serait autrement non visible. Dans ces cas-là, les demandes de répression peuvent se transposer en circonstance aggravante et influencer la sévérité de la peine.

En ce qui concerne maintenant les demandes de clémence ou de modération, les tribunaux font preuve d'une ambivalence fonctionnellement équivalente. Si ces demandes ne sont pas perçues comme une menace à l'intégrité du processus de détermination de la peine, les tribunaux se montrent clairement plus réceptifs. En effet, les tribunaux canadiens semblent avoir développé une certaine tolérance à l'égard des discours non punitifs de la part des victimes. Dans les affaires Gabriel et Bullen, les soussignés décrivent ces situations comme des « exceptions », mais nous préférons les présenter comme des « conditions de possibilités » pour la réception des recommandations des victimes sur la peine par le système judiciaire, recommandations qui, on l'aura compris, sont normalement à proscrire :

Recommendations as to penalty must be avoided, absent exceptional circumstances, i.e. a court-authorized request, an aboriginal sentencing circle, or as an aspect of a prosecutorial submission that the victim seeks leniency for the offender which might not otherwise reasonably be expected in the circumstances ${ }^{47}$.

45 Par menace, il faut comprendre une interférence qui risque de porter atteinte au principe de proportionnalité de même qu'au principe d'individualisation des peines. Du point de vue du droit, les victimes qui demandent de lourdes peines, de même que le public et l'opinion publique représentent un tel risque (Garcia et Dubé 2017b).

$46 \quad$ R. c. M.(C.A.), 19961 R.C.S. 557.

47 R. v. Gabriel, 1999, par. 33; italiques ajoutés. 
Victims have been allowed to express their views about the offender and about the sentence in conferences, circles and in other restorative justice sentencing processes [...]. Victims have also been allowed to comment about the offender and the sentence when both counsels agree ${ }^{48}[\ldots]$ and, curiously, when victims are supportive of the offender [...]. These practices are exceptions to the clear line courts have drawn preventing victims from commenting on the offender or on an appropriate sentence $e^{49}$.

Lorsque nous observons comment ces demandes de clémence se transposent en information dans la détermination de la peine, nous pouvons voir que celles-ci ont un poids marginal. En effet, les demandes de clémences des victimes sont généralement transposées en circonstance atténuante. Les énoncés suivants sont tirés de l'affaire Perry (2011) qui implique une course de rue (en motocyclette) ayant causé la mort de l'un des motocyclistes. Alors que le condamné a ralenti pour se ranger en bordure de route, la victime a poursuivi la course et est entrée en collision avec une voiture venant en sens inverse. Le condamné est reconnu coupable d'une conduite dangereuse causant la mort (Art. 249(4), C.cr.). Lors des audiences sur la détermination de la peine, la mère de la victime soumet une lettre au tribunal dans laquelle elle exprime sa position par rapport aux événements :

[...] Mes sentiments comme mère de la victime est que seul mon fils Éric est responsable de sa mort et personne ne me convaincra du contraire. Je demande donc par la présente la clémence de la cour pour Kevin Perry ${ }^{50}$.

Face à cette demande de clémence, le tribunal réagit de la manière suivante :

Le Tribunal précise avec respect qu'il n'appartient pas aux victimes ou à leur famille de qualifier si l'acte posé est criminel ou accidentel comme il appartient seulement aux tribunaux d'imposer les peines, même si de leur part s'y trouve un appel à la clémence. [...] Par contre, le Tribunal doit tenir compte des sentiments vécus par la mère de la victime décédée. Lorsque les membres de la famille d'une victime réclament dans leur douleur au tribunal une peine sévère, ce dernier doit prononcer une sanction dont un des facteurs aggravants sera les conséquences vécues par la famille. Le contraire s'appliquera aussi dans une situation comme la présente, lorsque malgré la douleur vécue par une mère qui perd son fils, elle fait appel à la clémence du Tribunal. Mais en aucun cas le fardeau d'imposer une peine ne doit reposer sur les épaules d'une victime ou de son entourage familial. Seuls, les tribunaux ont pour tâche de punir un individu conformément à la Loi et la jurisprudence ${ }^{51}$.

Même si, dans Perry, nous pouvons remettre en question le rapport du condamné avec la conséquence funeste de la mort de son ami, le tribunal a pu éviter le recours à l'incarcération en favorisant le sursis, et ce malgré une contrainte

48 Nous ne sommes pas parvenus à observer une situation dans laquelle le procureur de la couronne et l'avocat de la défense se sont mis d'accord pour laisser la victime se prononcer sur la peine. Nous croyons que ces négociations sont difficiles à observer dans les décisions. Cependant, cela ne signifie pas qu'elle est moins importante que les autres. Au contraire, il s'agit d'une condition de possibilité au même titre que les autres, mais que nous n'avons tout simplement pas pu observer dans notre matériel empirique.

R. c. Perry, 2011, par. 248-249; italiques ajoutés. 
sociojuridique qui l'empêchait d'y recourir ${ }^{52}$. Cependant, en aucun cas le tribunal ne s'appuie sur la demande de clémence de la mère du défunt pour valoriser le sursis. Toute l'argumentation repose sur la valorisation du principe d'individualisation de la peine ${ }^{53}$.

La demande de la mère du défunt n'est pas exclue du processus de détermination de la peine, mais son intégration subit une mise en forme sémantique : elle devient une demande de "clémence » et prend la forme d'une simple circonstance atténuante ${ }^{54}$; une circonstance parmi d'autres. Pour la mère de la victime, c'est le prix à payer pour être entendue par le tribunal. Dubé et Garcia (2017a, 14) décrivaient cette réaction des tribunaux comme une " reconversion [d'une attente a priori inaudible] en attente audible dotée d'une certaine capacité de connectivité minimale à l'intérieur de la cohérence interne du système ". L'énoncé provenant de l'affaire Perry, lorsque nous le faisons dialoguer avec les énoncés tirés de Gabriel et de Bullen sur les conditions de possibilités, tend à suggérer que la réception des demandes de clémence que nous venons de décrire constitue une tendance forte : ces demandes se transposent essentiellement en circonstance atténuante et peuvent influencer la sévérité de la peine (Labonté 2017). Par conséquent, même si les tribunaux font preuve d'écoute aussi à l'égard des demandes de clémence des victimes, cette écoute, à l'intérieur de la rationalité pénale moderne, ne bénéficie que d'une marge de manœuvre limitée.

Notre empirie a pu nous révéler une condition de possibilité supplémentaire qui autorise la victime à se prononcer sur la peine. Il s'agit des situations particulières où la peine juste et adéquate imposée ou en voie d'être imposée cause un préjudice additionnel à la victime ${ }^{55}$. Ce préjudice additionnel, qu'il se soit concrétisé ou non, peut justifier une peine plus clémente ou plus sévère. Dans ces situations, ce sont souvent les victimes elles-mêmes qui informent le tribunal des conséquences de la peine envisagée ou qui a été imposée. Nous avons pu observer ces situations dans les affaires Deschênes ${ }^{56}$ et Basque . $^{57}$.

L'affaire Deschênes porte sur une conduite avec les facultés affaiblies ayant causé des lésions. La victime et l'accusé, de bons amis, étaient à bord de la même voiture. La victime a perdu l'usage de ses mains et de ses jambes. L'accusé, quant à lui, s'en tire avec des blessures mineures et va agir comme un aidant naturel auprès de la victime durant les deux années qui s'écoulent entre la commission de l'infraction et les audiences de détermination de la peine. Lors des audiences sur la peine, la

52 Il s'agit du projet de loi de 2007 qui interdisait de recourir à l'emprisonnement avec sursis pour les infractions de courses de rues causant des lésions corporelles et la mort.

53 En 2013, la peine d'emprisonnement avec sursis est invalidée par la Cour d'appel et remplacée par une peine d'emprisonnement.

54 Il en va de même pour les pardons des victimes. Lorsque la victime pardonne les gestes de l'accusé, cette attitude miséricordieuse est réduite à une circonstance atténuante, peu importe le sens du pardon pour la victime. Que la victime pardonne en gage d'un arrêt des procédures ou pour son bien-être psychologique, le système judiciaire ne tirera de cet input qu'une simple circonstance atténuante.

55 Du côté du droit criminel anglais, Doak $(2008,150)$ observait également cette prise en compte de l'opinion de la victime sur la peine lorsque cette dernière aggravait la détresse de la victime.

56 R. c. Deschênes, 2012 QCCQ 10546 (canlii). Pour une analyse plus détaillée de cette décision, voir Labonté (2017).

57 R. v. Basque, 1999 CanLII 9448. 
victime réalise que son ami, l'accusé, risque une lourde peine de prison et s'adresse au tribunal pour lui demander de faire preuve de clémence. La victime informe le tribunal qu'une peine d'emprisonnement allait lui causer un préjudice additionnel en le privant de l'aide apportée par l'accusé. Le tribunal va faire preuve d'ouverture ${ }^{58}$ à l'égard de cette requête et, même s'il ne parvient pas à abandonner l'injonction d'une peine d'incarcération, il va prononcer une peine plus clémente, sous la fourchette établie par la jurisprudence : une peine d'emprisonnement discontinu. Notons que pour le tribunal, réduire la peine d'incarcération dans ce cas relève de la clémence, et non pas de la peine juste. Parler de «clémence " plutôt que d'une " peine juste " dans ce cas démontre encore une fois la profondeur de nos racines dans l'idéal punitif tel qu'il est valorisé par la rationalité pénale moderne.

Dans d'autres circonstances, le préjudice additionnel causé par la peine peut justifier l'augmentation de la sévérité d'une peine et favoriser l'affliction et l'exclusion sociale du condamné. C'est ce qui se produit dans l'affaire Basque où une employée d'un dépanneur a été victime d'un vol à main armée. Le juge de première instance a prononcé une peine d'emprisonnement avec sursis (purgée en collectivité). Le procureur a interjeté appel et le juge de deuxième instance a déterminé que la peine était trop clémente par rapport au geste causé. Il appuyait son raisonnement en partie sur la nécessité de dissuader et de dénoncer le comportement illégal, mais aussi sur le préjudice additionnel que le sursis à l'emprisonnement causait à la victime. Celle-ci avait informé le tribunal que le fait que l'accusé se trouvait en collectivité nuisait grandement à son bien-être psychologique et qu'elle aurait à déménager si le sursis devait être maintenu. La Cour d'appel a précisé que la justice ne devrait pas engendrer ce genre de situation pour la victime. Elle a alors remplacé l'emprisonnement avec sursis par une peine d'emprisonnement ferme.

Soulignons que, lorsque la peine est susceptible d'apporter un préjudice additionnel à la victime, la prise en compte de la demande victimaire semble avoir un impact beaucoup plus marqué sur le choix de la sentence que ne l'aurait une simple circonstance atténuante ou aggravante.

\subsection{La réception des attentes de closure}

Les attentes de closure peuvent " renvoyer à la fermeture (d'un dossier), au règlement (d'un cas) ou à la résolution (d'une affaire) " ou encore à " l'idée de mettre un terme à la souffrance » ${ }^{59}$ (Cauchie et Sauvageau 2013, 169). Cependant, il faut aussi distinguer entre ce que les tribunaux et les victimes associent au closure (Dubé et Garcia 2018, 9). En effet, cette distinction nous amène à observer une asymétrie sémantique du concept de closure.

58 Le juge va aller jusqu'à dire, dans le cas en l'espèce, que « la peine doit être modulée pour avantager la victime " et " [qu'il] y va de l'intérêt réel de la victime qui a convaincu le [tribunal] que si une peine de détention importante était imposée, la victime en subirait les conséquences " $(R . c$. Deschênes, 2012, par. 52 et 54)

59 Dans cette étude, les auteurs avaient limité leurs observations à des cas bien spécifiques, notamment à des cas d'infractions criminelles dont la conséquence a été la mort de la victime et où les peines sont les plus lourdes (Cauchie et Sauvageau, 2013, 169). En ce qui nous concerne, nous n'avons pas limité notre observation à ces cas précis. Nous avons plutôt élargi le contexte d'observation pour y inclure, notamment, les cas d'agression sexuelle, de viols, etc. 
Pour les victimes, le closure est plus susceptible de prendre le sens d'un «closure psychologique " qui, lorsqu'atteint, permet aux victimes et à leurs proches de continuer à vivre malgré les souffrances. Pour le système judiciaire, le closure prend plutôt le sens d'un «closure procédural » au sens d'un aboutissement des procédures judiciaires (ibid.). La détermination d'une peine, du point de vue du système, permet de fermer un dossier, de marquer un dossier comme étant réglé. Cependant, nous pouvons très bien imaginer qu'une victime continue de souffrir des conséquences du crime malgré la fin des procédures judiciaires. Nous pouvons également envisager la possibilité dans laquelle la peine prononcée crée un effet thérapeutique sur la victime et ses proches en leur permettant d'obtenir un closure psychologique. Il faut cependant faire preuve de prudence afin d'éviter de confondre, comme le soulignent Dubé et Garcia (2018, 9), l'effet avec la cause : l'abrègement des souffrances des victimes par le prononcé de la peine n'est pas un objectif de la peine admissible. Autrement dit, le système établit une distinction entre peine et thérapie $^{60}$. Il s'agit plutôt de situations qui dépendent de chaque victime et non d'une tâche que doivent remplir les peines. Du fait de cette distinction entre peine et thérapie, les attentes de closure psychologique ne parviennent à se stabiliser que sous la forme d'un closure procédural; le closure psychologique étant laissé aux victimes elles-mêmes.

Néanmoins, il peut arriver que les tribunaux se rapprochent du closure psychologique en cherchant à éviter de produire des souffrances additionnelles aux victimes, des souffrances qui seraient imputables au cours normal des procédures judiciaires (et non pas au crime subi). Comme nous l'avons vu avec Deschênes et Basque, lorsque la peine cause des torts supplémentaires à la victime, les tribunaux réagissent en cherchant à atténuer ce fardeau supplémentaire. Il en va de même pour les délais judiciaires : les longs délais et les incertitudes qu'ils génèrent pour les victimes sont reconnus comme ajoutant un préjudice à la victime $^{61}$. Toutefois, il ne faut pas se méprendre et conclure que les tribunaux cherchent à offrir un closure psychologique aux victimes et à leurs proches. Le rôle que les tribunaux se donnent par rapport au closure ne répond pas à une logique thérapeutique, mais plutôt à une logique de « justice » qui ne se détourne pas des pratiques punitives préétablies. Ils cherchent à éviter que le cours normal de la justice criminelle entraîne des souffrances additionnelles dues à l'injustice que représentent les longs délais judiciaires pour toutes les parties du conflit. Seulement, pour les victimes, les souffrances découlant des délais judiciaires s'ajoutent aux souffrances qu'elles ont déjà subies par l'intermédiaire de l'infraction. L'abrègement des souffrances générées par l'infraction continue d'être exclu en tant qu'objectif de la peine.

60 Les tribunaux reconnaissent et attribuent à la déclaration de la victime, et non pas à la peine, la possibilité d'engendrer un tel effet thérapeutique. Certaines recherches ont démontré que la soumission d'une déclaration engendrait ce type d'effet chez la victime (Manikis, 2015a, 90-91; Manikis, 2015b, 174).

61 Voir l'arrêt R. c. Jordan, 20161 R.C.S. 631, aux pages 644 et 650. 


\section{Conclusion : l'intégration des victimes dans la détermination de la peine représente-t-elle un obstacle à l'évolution du droit criminel moderne?}

Que pouvons-nous dire sur la relation entre le droit criminel et les victimes à partir de nos observations? D’abord, nous pouvons affirmer que le cadre pénal affecte la forme que prennent les attentes victimaires à l'intérieur des trames discursives du droit criminel dans le contexte de la détermination de la peine. Lorsque les victimes demandent à être protégées ou à obtenir une certaine tranquillité d'esprit, le programme de la RPM suggère certaines solutions au détriment d'autres, dont le recours aux sanctions privatives de liberté ou à certaines conditions lorsqu'une sanction en milieu ouvert est privilégiée. Ce lien s'observe également dans l'intégration difficile des demandes de clémence par les victimes, sauf lorsque la peine cause un préjudice additionnel à la victime. Dans cette circonstance, ce type de demande peut amener les tribunaux à aller dans une direction vers laquelle ils n'ont pas l'habitude d'aller : la modération et la clémence. Cependant, même dans ce contexte particulier, cette demande de clémence est insuffisante pour abandonner la logique punitive.

Ensuite, à la lumière de notre analyse, nous pouvons observer une volonté juridique de tenir compte de certaines attentes victimaires lorsque les conditions le permettent. Cette volonté juridique dissimulerait une forme de reconnaissance que les tribunaux canadiens auraient institutionnalisée au fil du temps, du fait de la présence marquée des victimes dans les procédures judiciaires (comme ailleurs en occident). Cependant, il ne faut pas se méprendre, cette reconnaissance n'a pas préséance sur la logique punitive préétablie du droit criminel. Au contraire, nos observations suggèrent plutôt que ce soit la première qui soit déterminée par la dernière. C'est à l'intérieur de la logique punitive interne du système que s'opère cette reconnaissance à partir de la considération des attentes victimaires, notamment par la reconnaissance des torts subis lors de l'évaluation de la gravité de l'infraction.

Nous pouvons faire dialoguer cette volonté juridique de combler certaines des attentes des victimes avec le paradigme de l'indépendance judiciaire du " juge gardien des promesses dans la cité » identifiée par Garcia $(2016,253)$. Ce paradigme s'oppose à celui du juge dans sa « tour d'ivoire » qui lui offrirait une position de surplomb sur les enjeux sociaux et une neutralité qui le protégerait des influences de la société civile (Ibid., 252). Si, autrefois, les liens étroits entre la cour et la société civile étaient perçus comme une menace à l'indépendance judiciaire, "cette proximité » serait devenue « la condition même de l'objectivité qu'exige l'indépendance judiciaire " (Garcia 2016, 253). Dans ce paradigme du « juge gardien des promesses dans la cité ", nous dit l'auteure, "les magistrats eux-mêmes seront les premiers à reconnaître que le temps est venu de créer des liens plus étroits avec la société civile et de développer une proximité plus réelle avec la communauté » (Ibid.). Ainsi, le rapprochement entre les victimes et le judiciaire ne serait qu'une expression particulière de cette récente manière d'envisager l'indépendance judiciaire.

Si cette proximité avec les victimes n'est désormais plus perçue comme une menace à l'indépendance judiciaire, mais bien comme l'une de ses conditions de possibilité, cette proximité risque-t-elle d'aggraver le problème de «non-évolution » (Pires 2002) du droit criminel? À cette question, notre exploration au sein de la jurisprudence canadienne nous permet de fournir quelques éléments de réponse. 
Par évolution, il faut comprendre ici une évolution identitaire, c'est-à-dire le passage d'un droit criminel qui s'auto-décrit comme étant "guerrier » à un droit criminel plus « citoyen » (Pires 2001b) et " responsif » (Nonet et Selznick 1978; Dubé et Labonté 2016). Pires (2001a, 200) précisait que, pour que l'intégration du public se fasse de manière à favoriser l'évolution du droit criminel, elle « doit être accompagnée d'un mode de pensée alternatif, tant sur le plan cognitif que de l'autoorganisation normative du système, ce qui est un phénomène aussi souhaitable que rare ». Il en va de même concernant l'intégration des victimes et de leurs attentes dans le processus de détermination de la peine. L'enjeu pour l'évolution du droit criminel résiderait moins dans l'intégration des victimes que dans la valorisation d'idées innovatrices pour fonder l'intervention du droit criminel. Une telle transformation impliquerait une volonté juridique de réduire considérablement le recours aux idées fondatrices de la rationalité pénale moderne au profit d'idées plus positives et inclusives comme celles valorisant le lien social entre l'accusé, la victime et la communauté ou encore celles valorisant une éthique " pour-l'autre » (Dubé et Labonté 2016, 696). Ce n'est que lorsque les praticiens du droit oseront s'affranchir des idées de la rationalité pénale moderne qu'ils pourront penser autrement l'intervention du droit criminel en général et la pratique sociale de la détermination de la peine en particulier.

\section{Références}

Adam, C., J.-F. Cauchie, M.-S. Devresse, F. Digneffe et D. Kaminski. 2014. Crime, justice et lieux communs. Bruxelles : Éditions Larcier.

Baril, M., M.-M. Cousineau et S Gravel. 1984. Mais nous les témoins... Une étude exploratoire des besoins des témoins au Palais de justice de Montréal. Collection Victimes d'actes criminels, document de travail no. 10. Ottawa : Ministère de la Justice.

Bensussan, J. 2009. Quelques réflexions sur un barbarisme juridique : la place de la victime dans le procès pénal. Dans La place de la victime dans le procès pénal, dir. Y. Strickler, 33-36. Bruxelles : Bruylant.

Boisvert, A.-M., et A. Jodouin. 2002. De l'intention à l'incurie : le déclin de la culpabilité morale en droit pénal canadien. Revue Générale de Droit 32:759-795.

Cartuyvels, Y., et L. Van Campenhoudt. 2004. Comment étudier les attentes des citoyens à l'égard de la Justice? Dans Public Opinion and the Administration of Justice. Popular Perceptions and Their Implications for Policy-Making in Western Countries, dir. S. Parmentier, G. Vervaeke, R. Doutrelepont et G. Kellens, 33-49. Bruxelles : Politeia.

Cassell, P. G. 2015. Introduction : the Maturing Victims' Rights Movement. Ohio State Journal of Criminal Law 13 (1): 1-4.

Cauchie, J.-F., et J. Sauvageau. 2013. La montée du closure : un allié ou un trublion pour la rationalité pénale moderne? Dans La rationalité pénale moderne: Réflexions théoriques et explorations empiriques, dir. R. Dubé, M. Garcia et M. R. Maìra, 167-191. Ottawa : Les Presses de l'Université d'Ottawa.

Doak, J. 2008. Victim's Rights, Human Rights and Criminal Justice: Reconceiving the Role of Third Parties. Oxford and Portland, Oregon : Hart Publishing.

Dubé, R. 2017. L'évolution des systèmes sociaux : comment penser la contribution des systèmes psychiques? Dans Systèmes psychiques et systèmes sociaux, dir. L. K. Sosoe, 78-105. Hildesheim : Georg Olms Verlag. 
Dubé, R., et J.-F. Cauchie. 2007. Enjeux autour de l'évolution du droit criminel moderne: quand les variations de la périphérie défient l'autorité redondante du centre. Déviance et Société 31 (4): 465-485.

Dubé, R., et M. Garcia. 2017a. Le judiciaire, la détermination de la peine et la polycontexturalité de l'opinion publique. Revue canadienne Droit et Société, 32 (1): 1-20.

Dubé, R., et M. Garcia. 2017b. La construction politique des attentes victimaires dans les débats parlementaires entourant la création de la loi pénale. Champ pénal/Penal field, 14:1-25. Récupéré sur http://champpenal.revues.org/9501

Dubé, R., et M. Garcia. 2018. Le juge et la victime : une nouvelle « union de faits » en droit criminel canadien? Document de travail inédit, 1-23.

Dubé, R. et S. Labonté. 2016. « Dénonciation, rétribution et dissuasion: repenser trois obstacles à l'évolution du droit criminel moderne ». Les Cahiers de droit, 57 (4): 685-713.

Duchastel, J. 1993. «Discours et informatique: des objets sociologiques?». Sociologie et Sociétés 25 (2): 157-170.

Elias, R. 1993. Victims Still : The political manipulation of crime victims. Newbury Park: Sage Publications.

Elias, R. 1986. The Politics of Victimization : Victims, victimology, and human rights. New York: Oxford University Press.

Erner, G. 2006. La société des victimes. Paris : La Découverte.

Garcia, M. 2013. La théorie de la rationalité pénale moderne : un cadre d'observation, d'organisation et de description des idées propres au système de droit criminel. Dans La rationalité pénale moderne : réflexions théoriques et explorations empiriques, dir. R. Dubé, M. Garcia et M. R. Machado, 37-72. Ottawa : Les Presses de l'Université d'Ottawa.

Garcia, M. 2016. Du juge « bouche de la loi » dans sa «tour d'ivoire » au juge "gardien des promesses » dans la cité. Canadian Criminal Law Review 21:237-267.

Garcia, M., et R. Dubé. 2017a. La réforme du droit criminel : une idée dont le temps est venu. Dans Réformer le droit criminel au Canada : défis et possibilité/Criminal Law Reform in Canada: Challenges and Possibilities, dir. J. Desrosiers, M. Garcia et M.-È. Sylvestre, 335. Montréal : Éditions Yvon Blais.

Garcia, M., et R. Dubé. 2017b. Une enquête théorique et empirique sur les menaces externes à l'indépendance judiciaire dans le cadre du sentencing. Revue générale de droit 47 (1): $5-45$.

Garland, D. 2001. The Culture of Control : Crime and social order in contemporary Society. Chicago: University of Chicago Press.

Grossman, M. G., et C. Kane. 2008. Victims of Crime and the Justice System. Dans Criminal Justice in Canada : A Reader, dir. M. G. Grossman et J. V. Roberts, 284-295. Toronto : Thomson Nelson.

Kaminski, D. 2009. Pénalité, management, innovation. Namur : Presses universitaires de Namur.

Katz, K. M. 2010. Opposing Scales of Justice: Victims' voice in the sentencing process. Canadian Criminal Law Review 14 (2): 181-231.

Kirchengast, T. 2010. Recent Reforms to Victim's Rights and the Emerging "Normative Theory of the Criminal Law." Criminal Law Quaterly 56:81-115.

Labonté, S. 2017. «La réception de la victime non punitive par le droit criminel au moment de déterminer la peine: une étude de cas ». Dans Réformer le droit criminel au Canada: défis et possibilités/Criminal Law Reform in Canada: Challenges and Possibilities, dir. J. Desrosiers, M. Garcia et M.-È. Sylvestre, 359-381. Montréal : Éditions Yvon Blais.

Lemonne, A. 2011. «L'existence d'une socialité vindicatoire? Tentative d'analyse à partir du discours des victimes ». Dans : "Justice! » Entre pénalité et socialité vindicatoire, 81-98. Ottawa : Laboratoire d'Études et de recherches sur la Justice, Université d'Ottawa. 
Manikis, M. 2015a. Victim Impact Statements at Sentencing: Towards a clearer understanding of their aims. University of Toronto Law Journal 65 (2): 85-123.

Manikis, M. 2015b. Imagining the Future of Victims' Rights in Canada : A comparative perspective. Ohio State Journal of Criminal Law 13 (1): 163-186.

Markin, T. 2017. Victim Rights in Sentencing: An examination of victim impact statements. Canadian Criminal Law Review 22:95-119.

Nonet, P., et P. Selznick. 1978. Law and Society in Transition: Toward a Responsive Law. New York : Octagon Books.

Parent, C. 1995. La déclaration de la victime au niveau de la sentence : une mesure adaptée aux besoins des victimes? Revue Générale de Droit 26:127-132.

Perrin, B. 2017. Victim Law: The Law of Victims of Crime in Canada. Toronto : Thomson Reuters Canada Limited.

Pires, A. P. 1990. Le devoir de punir : Le rétributivisme face aux sanctions communautaires. Revue canadienne de criminologie/Canadian Journal of Criminology 32 (3): 441-460.

Pires, A. P. 1997. "Échantillonnage et recherche qualitative ". Dans De quelques enjeux épistémologiques d'une méthodologie générale pour les sciences sociales, dir. J. Poupart, J.-P. Deslauriers, L.-H. Groulx, A. Laperrière, R. Mayer et A. P. Pires, 113-169. Québec: Gaëtan Morin Éditeur.

Pires, A. P. 1998. Aspects, traces et parcours de la rationalité pénale moderne. Dans Histoire des savoirs sur le crime et la peine. Tome II : La rationalité pénale et la naissance de la criminologie, dir. C. Debuyst, F. Digneffe, J.-M. Labadie et A. P. Pires, 3-51. De Boeck : De Boeck Université.

Pires, A. P. 2001a. La rationalité pénale moderne, la société du risque et la juridicisation de l'opinion publique. Sociologie et Sociétés 33 (1): 179-204.

Pires, A. P. 2001b. La « ligne maginot» en droit criminel : la protection contre le crime versus la protection contre le prince. Dans Il Diritto e La Differenza: Scritti in onore di Alessandro Baratta, dir. R. De Giorgi, vol. 1, 509-534. Lecce : Edizioni Pensa Multimedia.

Pires, A. P. 2002. Codifications et réformes pénales. Dans Crime et sécurité: l'état des savoirs, dir. L. Mucchielli et P. Robert, 84-92. Paris: La Découverte.

Pires, A. P., et F. Acosta. 1994. Les mouches et la bouteille à mouches : utilitarisme et rétributivisme classiques devant la question pénale. Revue Carrefour 16 (2): 8-39.

Quigley, T. 2010. The Dangers of Victim Impact Statements: A brief reply to Roberts and Manikis. Canadian Criminal Law Review 39 (15): 41.

Roach, K. 1999. Due Process and Victim's Rights: The new law and politics of criminal justice. Toronto : University of Toronto Press.

Roberts, J. V., et A. Edgar. 2006. La déclaration de la victime au moment de la détermination de la peine : expérience et perceptions des juges : un sondage réalisé dans trois provinces. Ottawa : Ministère de la justice.

Roberts, J. V., et M. Manikis. 2010. Victim Impact Statements at Sentencing: The relevance of ancillary harm. Canadian Criminal Law Rewiew 15:1-25.

Rossi, C. 2011. Droits-égards ou droits-devoirs? L'implication des proches des victimes d'homicide au sein des procédures pénales : conséquences et enjeux pour les personnes elles-mêmes. Dans «Justice! ». Entre pénalité et socialité vindicatoire, dir. F. Vanhamme, 149-169. Montréal : Érudit, coll. livres et actes. Consulté le 18 juin, 2018, sur http:// retro.erudit.org/livre/justice/2011/003013co.pdf

Salas, D. 2005. La volonté de punir : essai sur le populisme pénal. Paris : Hachette Littérature.

Verdun-Jones, S. N., et A. A. Tijerino. 2004. Four Models of Victim Involvement during Plea Negotiations: Bridging the gap between legal reforms and current legal practice. Canadian Journal of Criminology and Criminal Justice 46: 471-500. 


\section{Sébastien Labonté}

Wemmers, J.-A., et É. Raymond. 2011. La justice et les victimes : l'importance de l'information pour les victimes. Criminologie 44 (2): 157-169.

Young, A. N. 2001. Le rôle de la victime au sein du processus judiciaire une analyse bibliographique - 1989 à 1999. Ottawa : Ministère de la justice Canada.

\section{Sébastien Labonté}

Docteur en criminologie

Université d'Ottawa

slabo038@uottawa.ca 